\title{
INTRAGO: intraoperative radiotherapy in glioblastoma multiforme - a Phase I/II dose escalation study
}

\author{
Frank A Giordano ${ }^{1 *}$, Stefanie Brehmer ${ }^{2}$, Yasser Abo-Madyan ${ }^{1,3}$, Grit Welzel ${ }^{1}$, Elena Sperk ${ }^{1}$, Anke Keller ${ }^{1}$, \\ Frank Schneider ${ }^{1}$, Sven Clausen ${ }^{1}$, Carsten Herskind ${ }^{1}$, Peter Schmiedek ${ }^{2}$ and Frederik Wenz ${ }^{1}$
}

\begin{abstract}
Background: Glioblastoma multiforme (GBM) is the most frequent primary malignant brain tumor in adults. Despite multimodal therapies, almost all GBM recur within a narrow margin around the initial resected lesion. Thus, novel therapeutic intensification strategies must target both, the population of dispersed tumor cells around the cavity and the postoperative microenvironment. Intraoperative radiotherapy (IORT) is a pragmatic and effective approach to sterilize the margins from persistent tumor cells, abrogate post-injury proliferative stimuli and to bridge the therapeutic gap between surgery and radiochemotherapy. Therefore, we have set up INTRAGO, a phase I/II dose-escalation study to evaluate the safety and tolerability of IORT added to standard therapy in newly diagnosed GBM. In contrast to previous approaches, the study involves the application of isotropic low-energy (kV) $\mathrm{x}$-rays delivered by spherical applicators, providing optimal irradiation properties to the resection cavity.

Methods/Design: INTRAGO includes patients aged 50 years or older with a Karnofsky performance status of at least 50\% and a histologically confirmed (frozen sections) supratentorial GBM. Safety and tolerability (i.e., the maximum tolerated dose, MTD) will be assessed using a classical $3+3$ dose-escalation design. Dose-limiting toxicities (DLT) are wound healing deficits or infections requiring surgical intervention, IORT-related cerebral bleeding or ischemia, symptomatic brain necrosis requiring surgical intervention and early termination of external beam radiotherapy (before the envisaged dose of $60 \mathrm{~Gy}$ ) due to radiotoxicity. Secondary end points are progression-free and overall survival.
\end{abstract}

Trial registration: The study is registered with clinicaltrials.gov, number: NCT02104882 (Registration Date: 03/26/2014).

\section{Background}

Despite recent advances in therapy, Glioblastoma multiforme (GBM) is a lethal disease in most cases with a relatively short overall survival of roughly 15 months $[1,2]$. In virtually all cases, GBM recur locally within a narrow margin $(2-3 \mathrm{~cm})$ around the tumor cavity [3-5]. Although GBM are highly invasive and able to migrate along pre-existing structures such as blood vessels or white matter tracts [6,7] most (if not all) recurrent tumors paradoxically grow in close proximity to the resection margin [8]. Thus, though novel surgical techniques (such as fluorescence-guided resection) may have improved the

\footnotetext{
* Correspondence: frank.giordano@umm.de

'Department of Radiation Oncology, University Medical Center Mannheim, University of Heidelberg, Theodor-Kutzer-Ufer 1-3, 68167 Mannheim, Germany

Full list of author information is available at the end of the article
}

rates of macroscopic complete resections [9], and advanced radiotherapy techniques are at hand, no single or combined approach is sufficient to deplete microscopically dispersed tumor cells around the tumor cavity.

One of the techniques employed to tackle this challenging feature of GBM is intraoperative radiotherapy (IORT). IORT allows the delivery of high doses of electrons (IOERT) or low energy $x$-rays to the tumor bed while the surrounding healthy tissue is spared from radiation due to steep dose gradients [10]. This could lead to instant sterilization of the cavity surface from remaining tumor cells and delayed, or impaired, tumor cell proliferation between surgery and adjuvant therapies in deeper areas receiving lower doses. Furthermore, high single doses may elicit local (tumor bed) and systemic (immunogenic) responses which are not observed in this extent after conventionally fractionated radiotherapy $[11,12]$. 
Several, mostly small retrospective single-institutional studies have shown efficacy of the approach (reviewed in [13]). However, those pioneering studies were using IOERT and thus faced classical technical challenges that come along with a forward-scattering irradiation system in a setting with cylindrical or spherical tumor cavities. Postoperative dose reconstructions from the Munster University group demonstrated that many patients receiving IOERT exhibited areas of inadequate coverage (due to inaccurately selected electron energies, inappropriate cone sizes or angle errors). As expected, they found that median and 2-year survival significantly improved with better coverage (MS: 15.2 with adequate coverage vs. 9.3 months with inadequate coverage; 2year survival: $9.3 \%$ vs. $0 \%, \mathrm{p}=0.02)[14,15]$.

In contrast to the previously used forward-directed electron beams, the isotropic low-energy X-ray irradiation source used in the TARGIT (TARGeted Intraoperative radioTherapy) trial for breast cancer (INTRABEAM system) [16] is specifically attractive in IORT for brain tumors, where post-resection cavities are normally of complex shape. IORT using such spherical applicators has been proven feasible in pediatric [17] and adult primary brain tumors $[18,19]$. However, as there are no prospective data on safety and tolerability of IORT, we have designed INTRAGO, a phase I/II study built on the experience from past trials in several of which proof-of-principle has been demonstrated.

\section{Objectives}

\section{Primary objectives}

The primary goal is the definition of the maximal tolerated dose (MTD) of spherical isotropic kV-IORT in the setting of primary GBM.

\section{Secondary objectives}

Secondary endpoints are progression-free (PFS) and overall survival (OS).

\section{Methods/Design}

INTRAGO resembles a prospective, open-label, singlearm dose-escalation study (Figure 1). Dose escalation will be conducted in a "classical" $3+3$ manner with three patients entering each dose level. The decision to escalate to the next dose level is based on safety assessments after all patients of a cohort have reached month 3 (90 days) after IORT: If no dose-limiting toxicities (DLTs) occur in a cohort of 3 patients, the next cohort of 3 patients will be treated at the next higher dose level. If one of the 3 patients in a cohort experiences a DLT, an additional cohort of 3 patients will be treated at the same dose level: If no DLT occurs in the additional cohort, dose escalation will continue at the next higher dose level. Should one or more of the 3 patients in the

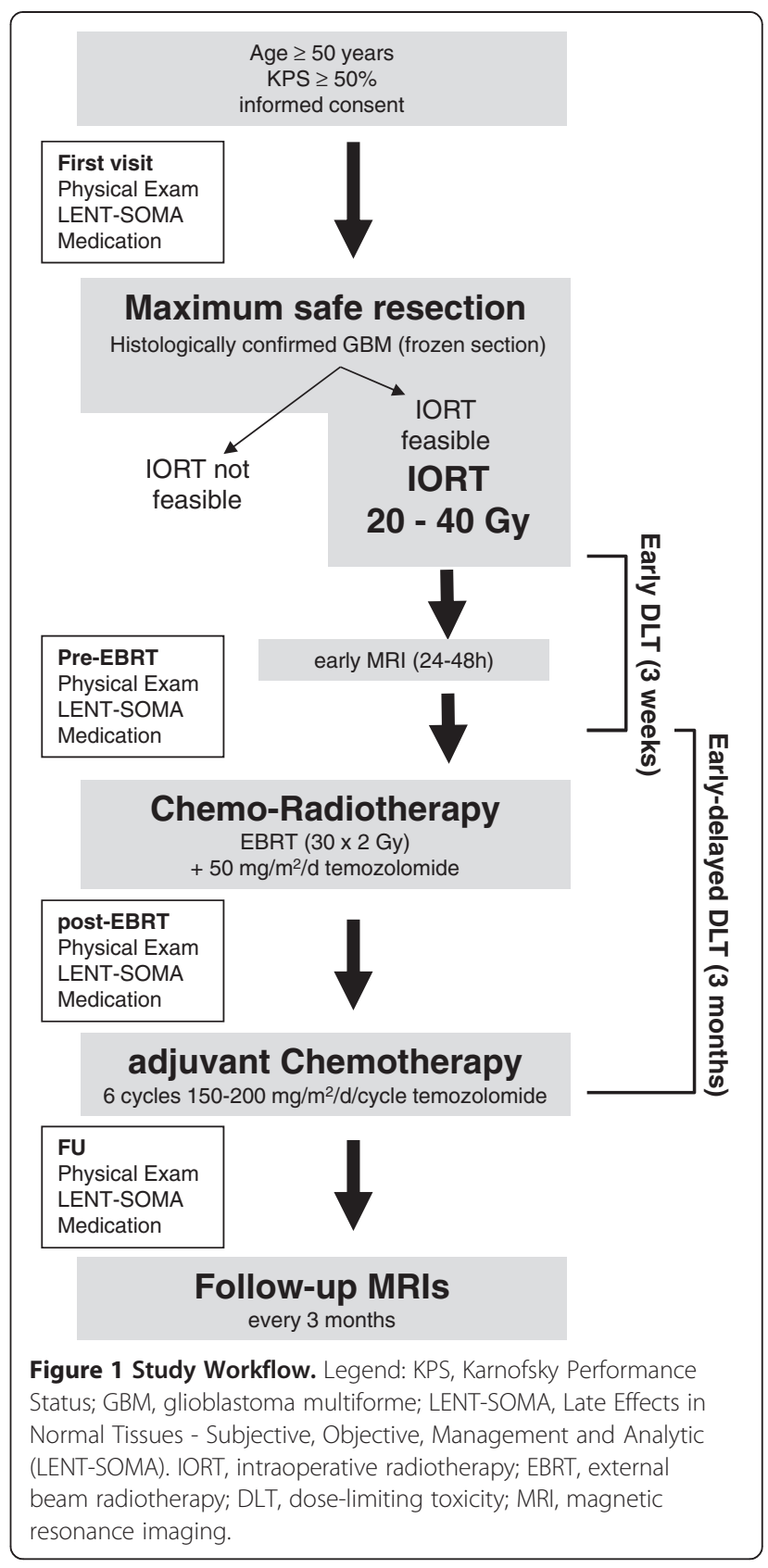

additional cohort experience a DLT, the MTD is considered exceeded and dose escalation will stop. The preceding dose level will then be considered as the MTD. If two or all of the 3 patients in a cohort experience DLTs, the MTD will be considered exceeded and the preceding dose level will be defined as the MTD. If only a total of 3 patients were treated at the potential MTD level, the potential MTD needs to be confirmed by recruiting an additional cohort of 3 patients: If none or one of the 6 patients experience a DLT, the MTD will be considered confirmed. If two or more patients experience a DLT, the next lower dose level will become the potential MTD. If 
no DLT occurs at all, the highest dose level will be defined as the MTD. Thus, the MTD is defined as the highest dose at which one or no DLT will have been observed among 6 patients.

\section{Patient selection}

Patients aged 50 years or older with a Karnofsky performance status (KPS) of at least 50\% and a histologically confirmed (frozen sections) supratentorial unifocal GBM are included. The tumor location should allow maximum safe resection. However, patients with tumors that are likely to be only partially removable are also eligible for the study.

\section{Inclusion criteria}

- Histologically confirmed glioblastoma multiforme in frozen sections

- Age $\geq 50$ years

- Karnofsky Performance Index $\geq 50 \%$

- Written Informed consent

- Adequate birth control (e.g., oral contraceptives)

\section{Exclusion criteria}

- Astrocytoma $\leq \mathrm{WHO}$ grade III

- Gliomatosis cerebri

- Multifocal lesions

- Infratentorial localization

- Previous cranial radiation therapy (any location)

- Uncontrolled intercurrent illnesses including, but not limited to, ongoing or active infection or psychiatric illness/social situations that would limit compliance with study requirements.

- Contraindications for general anaesthesia

- Bleeding or clotting disorders

- Contraindications for MRI or CT scans

- Pregnant or breastfeeding women

\section{Assessment of the primary objective (Safety)}

The primary goal is to determine the MTD, which will be assessed on the basis of pre-defined dose-limiting toxicities (DLT).

Two types of DLT are defined:

1) Early DLTs ( $\leq 3$ weeks after IORT):

- wound infections/wound healing difficulties requiring surgical intervention

- IORT-related cerebral bleeding or ischemia

2) Early-delayed DLTs ( $\leq 3$ months after IORT):

- Symptomatic brain necrosis requiring surgical intervention

- Early termination of EBRT (before the envisaged dose of 60 Gy) due to radiotoxicity
DLTs will be assessed on the basis of clinical presentation (physical examination, KPS, current medication), imaging studies (MRI) and on the basis of a neurological assessment using the Late Effects in Normal Tissues Subjective, Objective, Management and Analytic (LENT-SOMA) scales defined by the EORTC/RTOG $[20,21]$.

\section{Evaluation of DLT: clinical exams, medication}

Each follow-up visit has to include the most recent medical history, an inspection of the wound/scar and a thorough clinical exam. Episodes of partial or complex seizures must be documented. Wound healing (and the scar at FU) is followed with photo documentation. When performing a physical exam, there should be a specific emphasis on neurological functions. Specific awareness is advised for signs of cerebral edema (for example alterations in the level of consciousness, bradycardia, high blood pressure or inequality of pupillary size). All current medication and all changes made in the medication schedule have to be documented. Detailed information on doses (and dose changes) has to be only documented for corticosteroids and anticonvulsants.

\section{Evaluation of DLT: MRI}

Each follow-up visit includes MRI with contrast-enhanced (gadolinium) T1, axial T2 and axial T2-FLAIR sequences. Ischemic areas can be delineated using perfusion diffusionweighted imaging. It is challenging to distinguish early post-treatment blood-brain barrier permeability impairment from tumor recurrence and "true" brain necrosis. Here, methods such as Lesion Quotient (LQ), which resembles a ratio of the area delineated in a $\mathrm{T} 2$ sequence and the area of the corresponding contrast-enhanced T1 sequence may be applied [22]. In case MRI scans are inconclusive, positron emission tomography with amino acid tracers (such as ${ }^{18} \mathrm{~F}$-fluoro-ethyl-tyrosine) can be offered as preferential modality.

\section{Management of DLT}

Wound infections must be adequately treated, e.g. with dry absorbent dressing and, in case of positive wound swabs, systemic antibiotics should be administered matching to the antibiogram. In all cases of wound infection, blood workup (Complete blood counts, white blood counts, CRP) and imaging studies should be performed (CT or MRI) to rule out intracranial abscesses. Each case of wound infection (our healing difficulty) where conservative therapy fails and surgical revision is required is defined as DLT.

Due to the highly flexible positioning system of the IORT device, cases of cerebral ischemia or bleeding induced by the applicator are unexpected. Nevertheless, both were included into the protocol as DLTs and cases 
where (venous or arterial) ischemia or intracranial haemorrhage occur as a consequence of the IORT procedure (addition to the baseline risk), adequate therapy is required. Intracranial haemorrhages must be generally surgically removed if mass effects are exceeding the primary lesion volume. There is no symptomatic therapy for arterial or venous ischemia post resection. However, diffusion-weighted imaging (DWI) should be performed to document these events during follow-ups.

If radiation necrosis is suspected in MRI scans and no correlating clinical deterioration is noted, symptomatic therapy may not be necessary and observation is appropriate. For patients with mass effects or neurological symptoms, treatment options include conservative therapy with corticosteroids or anti-angiogenic substances (e.g., bevacizumab) or surgical debulking of the necrotic areas. The decision for either therapy should be made in interdisciplinary consensus (e.g., in interdisciplinary tumor boards). If surgery is required, the corresponding case will be defined as DLT.

All patients will undergo radiochemotherapy and will present five times per week at the linear accelerators. Complete blood counts (CBC), a chemistry panel, liver function tests (LFT) as well as renal function tests (RFT) are regularly performed to screen for hematotoxicity, hepatotoxicity or renal toxicity under temozolomide chemotherapy. Upon intolerance, incompliance or deterioration of CBCs, LFTs or RFTs, the therapy with temozolomide can be discontinued at any time point by any physician in charge. Any discontinuation of chemotherapy for more than 5 consecutive days has to be documented.

Cranial irradiation can elicit acute (side) effects occurring during treatment or early-delayed effects that appear within three months after radiochemotherapy [23]. In most cases, both acute and early delayed side effects largely resemble symptoms of mass effects (e.g., headache, nausea, worsening neurological symptoms), they are responsive to corticosteroids and they either gradually decrease in severity during daily radiotherapy (acute effects) or spontaneously resolve under cortisol (early-delayed). However, any case of radiotherapy-associated symptom deterioration that requires pausing of radiotherapy for more than 5 consecutive days has to be documented. If radiotherapy is entirely discontinued due to radiotherapy-associated side effects before the total dose of $60 \mathrm{~Gy}$ is reached, the case is considered to exhibit a DLT. As for chemotherapy, EBRT may be discontinued by any physician in charge.

\section{Assessment of secondary end points (efficacy)}

Secondary end points are progression-free (PFS) and overall survival (OS). PFS is defined as the interval (in days or months) from IORT to the date of first detection of progressive disease according to updated RANO criteria [24] or the date of last follow-up. The RANO working group recommended tight restrictions for evaluating progression within 12 weeks after radiotherapy as irradiation transiently increases the permeability of the (peri)tumoral vasculature which in turn impairs the validity of MRI scans during this period [25].

In INTRAGO, only cases where new T1-enhancing lesions are detected beyond the $80 \%$ isodose or new lesions that are histologically proven to be new manifestations of GBM are defined as true progression within a timeframe of 12 weeks after EBRT.

After this period, progressive disease is defined if one of the following RANO criteria applies:

- New T1-contrast-enhancing lesions outside of radiation field on decreasing, stable, or increasing doses of corticosteroids

- Increase by $\geq 25 \%$ in the sum of the products of perpendicular diameters between the first postradiotherapy scan, or a subsequent scan with smaller tumor size, and the scan at 12 weeks or later on stable or increasing doses of corticosteroids.

- Clinical deterioration attributable to tumor progress (and not to concurrent medication or diseases)

- Increased T2/FLAIR compared with baseline scan or best response after initiation of therapy on stable or increasing doses of corticosteroids.

OS is defined as the interval (in days or months) from IORT to the date of death from any cause.

\section{Imaging, interventions, follow-up}

First visit

Patients that are eligible for the study will be initially examined and the general medical condition (height, weight, general condition, Karnofsky index, medical history, medication) and the neurological (baseline) status (neurological exam and LENT-SOMA scales) will be assessed. All patients have to be willing and able to undergo repetitive MRI scans. Written informed consent must be obtained at least $24 \mathrm{~h}$ prior to surgery and IORT.

\section{Preceding imaging studies}

All patients enrolled will receive preoperative MRI imaging (minimum 1.5 T) including contrast-enhanced T1weighted magnetization-prepared rapid gradient echo (MP-RAGE) sequences to provide a 3D data set for image-guided surgery. Advanced imaging (such as perfusion or diffusion weighted imaging) may be performed at this stage but is not mandatory.

\section{IORT planning, risk structures}

The optic nerve(s) (or the chiasm, respectively) and the brain stem are defined as risk structures and have to be 
identified on pre-operative scans. If any risk structure is located $\leq 2 \mathrm{~cm}$ to a T1-enhancing lesion in pre-operative MRIs, intraoperative imaging (e.g. intraoperative ultrasound or in-room CT/MRI) should be used after removal of the tumor (to account for potential brain shifts) to document distances to the applicator surface and to allow dose definition.

\section{Surgery}

The resection procedure should be performed as image(neuronavigation) guided surgery with techniques that meet individual center standards and preferences. Resection techniques may include suction, bi-/monopolar cautery or ultrasound aspiration. Intraoperative application of 5-aminolevulinic acid (5-ALA) can be used to determine residual tumor tissue. A maximum safe resection approach is recommended, but not mandatory. Due to the possibility of liquor accumulation (or retention) around the applicator and subsequent lowering of doses to the target volume (i.e. the resection cavity wall), ventricular opening during surgery should be avoided whenever possible.

\section{Frozen section}

To establish the diagnosis of GBM, representative tissue samples have to be sent for histopathological examination. The frozen section/cryosection procedure can be performed according to local standards. In case histopathological hallmarks of grade 4 are present, the patient fulfils all inclusion criteria. If the diagnosis of GBM can not be reliably established with cryosection or if additional analyses are necessary, IORT must be omitted.

\section{Dose prescription and application of IORT}

Following establishment of the diagnosis via frozen section and after the surgeon defines the macroscopic (or 5-ALAdelineated) tumor to be satisfactorily removed, IORT will be prepared. All potentially involved risk structures that have been defined in pre-operative imaging (see above) may have displaced consequent to neuro-shifting (i.e. reduced intracerebral pressure and loss of liquor after resection) and should be re-identified with intraoperative imaging (ultrasound or in-room CT/MRI).

For the two risk structures (Optical nerve/chiasm and brain stem), dose constraints of 12 Gy (Optical nerve) and 12.5 Gy (brain stem) are commonly accepted in LINACbased EBRT according to the QUANTEC (Quantitative Analyses of Normal Tissue Effects in the Clinic) recommendations [26]. Since $\mathrm{kV}$-irradiation shows an increased relative biological effectiveness (RBE) [27], adapted dose constraints $\left(D_{\text {Max }}\right)$ of 10 Gy apply for both structures during IORT with the (kV-based) INTRABEAM-System. $D_{\text {Max }}$ to these structures are then defined intraoperatively on the basis of the dose-depth profiles of the corresponding applicator. If the doses to the risk structures at IORT
(D IORT) exceed 10 Gy, IORT is technically not feasible and has to be omitted (screening failure). If any risk structure is likely to receive a $\mathrm{D}_{\text {IORT }}$ of $>10$ Gy (e.g., the risk structure has direct contact to the applicator surface) it must be sufficiently shielded with cut-to-size tungsten-filed silicone shielding strips. Shielding with one layer tungstensilicone strips will reduce the $\mathrm{D}_{\text {IORT }}$ by $90 \%$.

Based on the cavity geometry and adjacent functional brain areas, the most suitable applicator will be chosen by the team of surgeons and radiation oncologists (sizes rage from $1.5-5.0 \mathrm{~cm}$ in $0.5 \mathrm{~cm}$ steps). The applicator will be inserted in the cavity correct positioning and adjacent risk structures will then be again visualized using intraoperative imaging (ultrasound or in-room CT/ MRI).

Fluids surrounding the surface should be ruled out or removed. The applicator is then taken out and mounted onto the INTRABEAM system. Next, the arm and the source are covered with the sterile drape and the mounted applicator is again fitted into the resection cavity. Radiation will then be initiated by a radiation oncologist for a defined time span as calculated by the machine software.

After IORT, surgery will be continued in a regular fashion without specific additional requirements.

\section{Radiation protection issues}

IORT has to be delivered in accordance with federal, state and/or local regulations on radiation protection. IORT with the INTRABEAM ${ }^{\circ}$ System does not require structural alterations if the operating room is approved for C-arm fluoroscopy [28].

\section{Early postoperative MRI}

Early postoperative MRIs must be performed within a window of 24-48 h after surgery and must be analyzed in a standardized assessment. Before contrast application T1-hyperintense lesions must be used to evaluate residual blood/heme and T2-TSE, T2-FLAIR, and DWI to evaluate ischemia. After contrast application, T1-hyperintense masses or nodules (residual tumor tissue) have to be quantified and the following has to be documented:

- Complete resection: removal of at least $98 \%$ of the T1-enhancing lesions.

- Subtotal resection: removal of $88-98 \%$ of the T1-enhancing lesions.

- Partial resection: removal of less than $88 \%$ of the T1-enhancing lesions.

\section{Pre-EBRT visit}

Before EBRT and concomitant chemotherapy is initiated, all patients will be re-examined (including a neurological exam, an update on medication and a re-assessment based on LENT-SOMA scales) to document changes 
with regard to the preoperative condition and to exclude contraindications for EBRT (such as impaired wound healing or active infection). Wound healing must be followed with photo documentation.

\section{External-beam radiotherapy (EBRT) treatment planning}

Contrast-enhanced T1- and T2-FLAIR sequences have to be co-registered with the planning CT scan for radiotherapy planning. The planning target volume (PTV) is defined as the peritumoral edema in FLAIR sequences plus a $2 \mathrm{~cm}$ margin. Lenses, retinae, optical nerves and/ or chiasm and brain stem have to be defined as organs at risk (OAR). The corresponding planning risk volumes (PRV) are defined as OAR plus a $3 \mathrm{~mm}$ safety margin. For all OAR, point dose (dose to a volume $>0.03 \mathrm{~cm}^{3}$ ) constraints apply (Table 1 ). In case an OAR was preirradiated during IORT (with or without shielding), the dose applied to the OAR during IORT ( $\mathrm{D}_{\text {IORT }}$ ) has to be converted into an dose that is equivalent to a 2 Gy fractionation scheme (EQD2) using an $\alpha / \beta$ ratio of 2 and considering an RBE of 1.5 as follows (Table 2 gives exemplary $\mathrm{D}_{\mathrm{IORT}}$ and EQD2 values):

$$
\begin{aligned}
\mathrm{EQD} 2 & =1.5 \cdot \mathrm{D}_{\mathrm{IORT}} \bullet\left(\mathrm{D}_{\mathrm{IORT}}+\alpha / \beta\right) /(2+\alpha / \beta) \\
& =1.5 \cdot \mathrm{D}_{\mathrm{IORT}} \bullet\left(\mathrm{D}_{\mathrm{IORT}}+2\right) / 4
\end{aligned}
$$

Adequate target volume coverage should be considered by ensuring that $95-107 \%$ of the prescribed dose is received by $95 \%$ of the PTV. Both, 3D-CRT or IMRT may be applied, beam energies must at least be $6 \mathrm{MV}$. A total dose of 60 Gy delivered in 30 fractions (2 Gy/ fraction) will be applied to the PTV. In case the PTV includes a PRV which would in consequence not receive a dose within the given constraints, a specific risk-organ PTV (PTV-R) has to be delineated to allow separate dose reconstruction.

\section{EBRT}

EBRT must be initiated within 4 weeks after surgery and IORT. All patients will receive five fractions of radiotherapy per week on a conventional linear accelerator (LINAC) with thermoplastic mask fixation. Pausing of radiotherapy is allowed for maximum 5 consecutive days. During EBRT, patients are routinely seen once to

Table 1 Point dose constraints (dose to a volume $>0.03 \mathrm{~cm}^{3}$ ) for OAR

\begin{tabular}{ll}
\hline Risk organ & Maximum point dose (EQD2) \\
\hline Lenses & $7 \mathrm{~Gy}$ \\
Retinae & $50 \mathrm{~Gy}$ \\
Optical nerves/chiasm & $55 \mathrm{~Gy}$ \\
Brain stem & $66 \mathrm{~Gy}$ \\
\hline
\end{tabular}

Table 2 Exemplary EQD2 values for various IORT doses ( $\left.D_{\text {IORT }}\right)$ to risk organs

\begin{tabular}{lr}
\hline $\mathbf{D}_{\text {IORT }}$ & EQD2 \\
\hline 3 & $6 \mathrm{~Gy}$ \\
4 & $9 \mathrm{~Gy}$ \\
5 & $13 \mathrm{~Gy}$ \\
6 & $18 \mathrm{~Gy}$ \\
7 & $24 \mathrm{~Gy}$ \\
\hline
\end{tabular}

twice weekly by physicians and evaluated for adverse reactions.

\section{Concomitant chemotherapy}

During EBRT, all patients will receive concomitant temozolomide-based chemotherapy with a total oral dose of $50 \mathrm{mg} / \mathrm{m}^{2} / \mathrm{d}$ five times per week (on radiotherapy treatment days). Chemotherapy will be initiated at the first day of radiotherapy and will be continued until the last day of radiotherapy. Discontinuation of chemotherapy is allowed for maximum 5 consecutive days.

\section{Adjuvant chemotherapy}

Four weeks after radiochemotherapy, adjuvant chemotherapy with temozolomide is initiated if the patients do not exhibit contraindications (low RBC, WBC and/or platelet counts, previous adverse reactions). The doses will be selected in accordance with the Stupp-protocol (150-200 $\mathrm{mg} / \mathrm{m}^{2} / \mathrm{d}$ temozolomide, day $1-5$ every 28 days) [2]. At least six cycles of chemotherapy should be applied. If a patient tolerates the cycling chemotherapy with temozolomide well, additional cycles may be applied.

\section{Post-EBRT follow-ups}

Follow-up visits after radiochemotherapy will be scheduled quarterly. Each visit is preceded by brain MRI imaging studies (contrast-enhanced T1, T2 and T2-FLAIR sequences). Advanced imaging (diffusion- and perfusionweighted sequences) is optional. The MRI scans are evaluated by a board-certified radiologist or neuroradiologist in accordance to updated RANO criteria (see above). Followup visits also include an assessment of the general medical condition (height, weight, general condition, Karnofsky index, medical history, medication) and the neurological status (neurological exam and LENT-SOMA scales).

\section{Serious adverse events}

Treatment-emergent (i.e. IORT-related) events that are fatal, life threatening or classified as LENT-SOMA grade IV toxicity resemble a serious adverse event (SAE) and have to be documented. In case of death an autopsy will be pursued. 


\section{Ethical aspects, trial registration}

INTRAGO is approved by the local ethics committee (Medical Ethics Commission II of the Faculty of Medicine Mannheim, University of Heidelberg; 2013-548SMA) and the Federal Office of Radiation Protection (Z 5-22462/2-2013-063). The trial is registered with clinicaltrials.gov, number: NCT02104882.

\section{Discussion}

We here present the first dose-finding study on lowenergy intraoperative radiotherapy for glioblastoma multiforme. Previous approaches used forward-directed electron beams that only inconsistently provided sufficient target volume coverage, leading to inconsistency in reported outcomes [15,29-37]. Within INTRAGO, the spherically irradiating devices of the INTRABEAM system are used to enable geometry-optimized IORT. This may for the first time enable sufficient dose delivery to the resection cavity and to remaining tumor cells.

On may argue that there is no benefit of further dose escalation after resection due to failure of trials involving dose escalation or additional radiosurgery to the tumor bed [38]. We challenge this conclusion on the basis of two facts that involve the (crucial) time between surgery and adjuvant therapy:

First, it is known that the mean doubling time of a GBM stem cell may be as fast as 24 hours and consequently, the waiting time for EBRT correlates with overall survival $[39,40]$. IORT is embedded in the surgical removal of the mass and thus will likely prevent and/or slow down this exceptionally fast tumor growth in this timeframe. Second, GBM growth shows dose-dependency as early studies showed that doses of at least 50 Gy are required to improve overall survival $[41,42]$. In the RTOG 98-03 trial, patients treated with 66 Gy showed the worst (11.6 months) and those receiving 84 Gy showed the best survival rates (19.3 months) without increased rates of toxicities [43]. Of note, this study was conducted with rather "old" techniques and in the era of more advanced functional imaging (such as PET) and irradiation techniques (intensity-modulated radiotherapy) dose-escalation may be even more safely and efficiently conducted [44]. We think that the reason why several other doseescalation trials were failing is likely to be related to processes occurring during the time between surgery and adjuvant therapy: any wounded site creates a specific stimulatory environment to promote healing, which inadvertently provides remaining cancer cells with strong pro-proliferative, pro-migratory and antiapoptotic stimuli $[45,46]$. In breast cancer, it has been shown that this unwanted response of the injured microenvironment can be attenuated with IORT [47]. This had direct consequences on the clinical outcome: patients that received IORT in a sequential operation, i. e. after the first surgery confirmed the diagnosis ('postpathology' cohort) showed higher local recurrence rates (5.4\%) compared with patients that received IORT during first surgery (2.1\%; 'pre-pathology' cohort) [16]. Whether or not traumatic brain injury has a similar strong influence on GBM cell proliferation post surgery as observed in breast cancer has not been demonstrated yet. However, as the injured brain is very well known to respond with an impressive cytokine cocktail that efficiently promotes astrocytic activation and proliferation [48-50], we believe that similar (and, regarding survival, likely highly beneficial) quenching of the tumor microenvironment may be achievable after IORT for GBM.

Symptomatic brain necrosis requiring surgical intervention was defined as DLT. Brain necroses appeared in multiple IOERT trials and they mostly correlated with improved survival [29]. This, together with the fact that bevacizumab is a novel and effective option to conservatively treat brain necroses [51] was prompting us to only consider brain necroses as a DLT if they become symptomatic and if they require surgery. However, although we do expect cases of brain necrosis, highly elevated rates are less likely as it is well known that the irradiated volume of brain is the key determinant for this side effect [52]. The device used for IORT in INTRAGO uses low-energy $(\mathrm{kV})$ photons that show exponential attenuation along their path [53]. Logically, the area receiving high(est) doses is a margin of maximum 1-1.5 cm width around the tumor cavity (IORT with 40 Gy surface dose at dose level III would result in doses of 12 Gy at $1 \mathrm{~cm}$, $8 \mathrm{~Gy}$ at $1.5 \mathrm{~cm}$ and $4 \mathrm{~Gy}$ in $2 \mathrm{~cm}$ depth), which is a volume that is eventually not large enough to become clinical apparent.

INTRAGO is the first prospective IORT study in the era of temozolomide. The alkylating agent has become a crucial part of standard treatment after the pivotal EORTC/NCIC study showed a considerable improvement of both overall and long-term survival rates if the substance is added to radiotherapy and given as adjuvant chemotherapy [1]. It is believed that the increased rates of blood-brain barrier permeability impairments that are seen after radiochemotherapy (and which are often misinterpreted as progressive disease) are predominantly caused by temozolomide $[25,54]$. This abnormal local reactions together with pre-clinical investigations point to additive and/or even synergistic activity of both modalities and it will be of specific interest to see whether IORT can further modulate these interactions $[55,56]$.

In conclusion, INTRAGO is the first dose-finding study on low-kV-IORT for newly diagnosed GBM in the temozolomide era with optimized geometry adaptation. It should provide a robust basis for subsequent randomized (phase II or III) trials, in which superiority over standard treatment must be tested. 


\section{Abbreviations}

3D-CRT: Three-Dimensional Conformal Radiation Therapy; CBC: Complete Blood Count; CT: Computer Tomography; DIORT: Dose applied to an OAR during IORT; DLT: Dose-Limiting Toxicity; DWI: Diffusion-Weighted (Magnetic Resonance) Imaging; EBRT: External Beam Radiotherapy; EORTC: European Organisation for Research and Treatment of Cancer; EOD2: Equivalent Dose in 2 Gy Fractions; FLAIR: Fluid Attenuated Inversion Recovery; FET: ${ }^{18}$ F-fluoroEthyl-Tyrosine; FU: Follow-Up; GBM: Glioblastoma Multiforme; IMRT: IntensityModulated Radiation Therapy; INTRAGO: Intraoperative Radiotherapy in Glioblastoma Multiforme; IOERT: Intraoperative Electron Radiotherapy; IORT: Intraoperative Radiotherapy; KPS: Karnofsky Performance Status; kV: Kilovoltage $\left(10^{3} \mathrm{~V}\right.$ ); LENT-SOMA: Late Effects in Normal Tissues - Subjective, Objective, Management and Analytic; LINAC: Linear Accelerator; LFT: Liver Function Tests; LQ: Lesion Quotient; MP-RAGE: Magnetization-Prepared Rapid Gradient Echo; MRI: Magnetic Resonance Imaging; MTD: Maximum Tolerated Dose; OAR: Organ at Risk; OS: Overall Survival; PET: Positron Emission Tomography; PFS: Progression-Free Survival; PTV: Planning Target Volume; QUANTEC: Quantitative Analyses of Normal Tissue Effects in the Clinic; RANO: Response Assessment in Neuro-Oncology; RBE: Relative Biological Effectiveness; RFT: Renal Function Tests; RTOG: Radiation Therapy Oncology Group; SAE: Serious Adverse Event; TARGIT: Targeted Intraoperative Radiotherapy (randomized phase III study); WBC: White Blood Cell Count.

\section{Competing interests}

Carl Zeiss Medical AG supports radiobiological research at UMM. FG receives travel grants and/or speaker's honoraria from Carl Zeiss Medical AG, Merck Serono $\mathrm{GmbH}$ and Roche Pharma AG. The authors declare that they have no competing interests.

\section{Authors' contributions}

FG, SB, FW, PS and FW designed the study; FG, SB, YAM, GW and FW wrote the protocol; FS, SC and CH contributed and reviewed radiobiological and physical aspects; ES and AK handled ethics and regulatory affairs; FG, SB, YAM and FW wrote the paper draft and all authors have contributed and approved the final version of the manuscript.

\section{Author details}

'Department of Radiation Oncology, University Medical Center Mannheim, University of Heidelberg, Theodor-Kutzer-Ufer 1-3, 68167 Mannheim, Germany. ${ }^{2}$ Department of Neurosurgery, University Medical Center Mannheim, University of Heidelberg, Mannheim, Germany. ${ }^{3}$ Department of Clinical Oncology and Nuclear Medicine (NEMROCK), Cairo University, Cairo, Egypt.

Received: 15 July 2014 Accepted: 16 December 2014

Published: 22 December 2014

\section{References}

1. Stupp R, Mason WP, van den Bent MJ, Weller M, Fisher B, Taphoorn MJ, Belanger K, Brandes AA, Marosi C, Bogdahn U, Curschmann J, Janzer RC, Ludwin SK, Gorlia T, Allgeier A, Lacombe D, Cairncross JG, Eisenhauer E, Mirimanoff RO, European Organisation for Research and Treatment of Cancer Brain Tumour and Radiation Oncology Groups, National Cancer Institute of Canada Clinical Trials Group: Radiotherapy plus concomitant and adjuvant temozolomide for glioblastoma. N Engl J Med 2005, 352(10):987-996.

2. Stupp R, Hegi ME, Mason WP, van den Bent MJ, Taphoorn MJ, Janzer RC, Ludwin SK, Allgeier A, Fisher B, Belanger K, Hau P, Brandes AA, Gijtenbeek J, Marosi C, Vecht CJ, Mokhtari K, Wesseling P, Villa S, Eisenhauer E, Gorlia T, Weller M, Lacombe D, Cairncross JG, Mirimanoff RO, European Organisation for Research and Treatment of Cancer Brain Tumour and Radiation Oncology Groups, National Cancer Institute of Canada Clinical Trials Group: Effects of radiotherapy with concomitant and adjuvant temozolomide versus radiotherapy alone on survival in glioblastoma in a randomised phase III study: 5-year analysis of the EORTC-NCIC trial. Lancet Oncol 2009, 10(5):459-466.

3. Wallner KE, Galicich JH, Krol G, Arbit E, Malkin MG: Patterns of failure following treatment for glioblastoma multiforme and anaplastic astrocytoma. Int J Radiat Oncol Biol Phys 1989, 16(6):1405-1409.

4. Gaspar LE, Fisher BJ, Macdonald DR, LeBer DV, Halperin EC, Schold SC Jr, Cairncross JG: Supratentorial malignant glioma: patterns of recurrence and implications for external beam local treatment. Int J Radiat Oncol Biol Phys 1992, 24(1):55-57.

5. Choucair AK, Levin VA, Gutin PH, Davis RL, Silver P, Edwards MS, Wilson CB Development of multiple lesions during radiation therapy and chemotherapy in patients with gliomas. J Neurosurg 1986, 65(5):654-658.

6. Scherer J: The forms of growth in gliomas and their practical significance. Brain 1940, 40:631-635.

7. Holland EC: Glioblastoma multiforme: the terminator. Proc Natl Acad Sci U S A 2000, 97(12):6242-6244.

8. Petrecca K, Guiot MC, Panet-Raymond V, Souhami L: Failure pattern following complete resection plus radiotherapy and temozolomide is at the resection margin in patients with glioblastoma. J Neurooncol 2013, 111(1):19-23.

9. Stummer W, Pichlmeier U, Meinel T, Wiestler OD, Zanella F, Reulen HJ: Fluorescence-guided surgery with 5-aminolevulinic acid for resection of malignant glioma: a randomised controlled multicentre phase III trial. Lancet Oncol 2006, 7(5):392-401.

10. Calvo FA, Meirino RM, Orecchia R: Intraoperative radiation therapy first part: rationale and techniques. Crit Rev Oncol Hematol 2006, 59(2):106-115.

11. Veldwijk MR, Zhang B, Wenz F, Herskind C: The biological effect of large single doses: a possible role for non-targeted effects in cell inactivation. PLOS One 2014, 9(1):e84991.

12. Herskind C, Wenz F: Radiobiological aspects of intraoperative tumour-bed irradiation with low-energy X-rays (LEX-IORT). Translational Cancer Research 2014, 3(1):3-17.

13. Giordano FA, Abo-Madyan Y, Brehmer S, Herskind C, Sperk E, Schneider F, Clausen S, Welzel G, Schmiedek P, Wenz F: Intraoperative radiotherapy (IORT) - a resurrected option for treating glioblastoma? Translational Cancer Research 2014, 3(1):94-105.

14. Schueller P, Palkovic S, Moustakis C, Kónemann S, Wassmann H, Willich N: Clinical results and isodose planning of neuronavigation-guided intraoperative radiotherapy (IORT) in 77 brain tumor patients: adequate target volume coverage improve results. Rev Cancer (Madrid) 2008, 22(extra):1-58.

15. Schueller P, Micke O, Palkovic S, Schroeder J, Moustakis C, Bruns F, Schuck A, Wassmann $\mathrm{H}$, Willich $\mathrm{N}$ : 12 years' experience with intraoperative radiotherapy (IORT) of malignant gliomas. Strahlenther Onkol 2005, 181(8):500-506.

16. Vaidya JS, Wenz F, Bulsara M, Tobias JS, Joseph DJ, Keshtgar M, Flyger HL, Massarut S, Alvarado M, Saunders C, Eiermann W, Metaxas M, Sperk E, Sütterlin M, Brown D, Esserman L, Roncadin M, Thompson A, Dewar JA, Holtveg HM, Pigorsch S, Falzon M, Harris E, Matthews A, Brew-Graves C, Potyka I, Corica T, Williams NR, Baum M, TARGIT trialists' group: Risk-adapted targeted intraoperative radiotherapy versus whole-breast radiotherapy for breast cancer: 5 -year results for local control and overall survival from the TARGIT-A randomised trial. Lancet 2014, 383(9917):603-613.

17. Kalapurakal JA, Goldman S, Stellpflug W, Curran J, Sathiaseelan V, Marymont $\mathrm{MH}$, Tomita T: Phase I study of intraoperative radiotherapy with photon radiosurgery system in children with recurrent brain tumors: preliminary report of first dose level (10 Gy). Int J Radiat Oncol Biol Phys 2006, 65(3):800-808

18. Takakura K, Kubo O: Treatment of malignant brain tumors. Gan To Kagaku Ryoho 2000, 27(Suppl 2):449-453.

19. Lyons $M$, Phang I, Eljamel S: The effects of PDT in primary malignant brain tumours could be improved by intraoperative radiotherapy. Photodiagnosis Photodyn Ther 2012, 9(1):40-45.

20. Pavy JJ, Denekamp J, Letschert J, Littbrand B, Mornex F, Bernier J, GonzalesGonzales D, Horiot JC, Bolla M, Bartelink H: EORTC Late Effects Working Group. Late effects toxicity scoring: the SOMA scale. Radiother Oncol 1995, 35(1):11-15.

21. Pavy JJ, Denekamp J, Letschert J, Littbrand B, Mornex F, Bernier J, GonzalesGonzales D, Horiot JC, Bolla M, Bartelink H: EORTC Late Effects Working Group. Late Effects toxicity scoring: the SOMA scale. Int J Radiat Oncol Biol Phys 1995, 31(5):1043-1047.

22. Dequesada IM, Quisling RG, Yachnis A, Friedman WA: Can standard magnetic resonance imaging reliably distinguish recurrent tumor from radiation necrosis after radiosurgery for brain metastases? A radiographic-pathological study. Neurosurgery 2008, 63(5):898-903. discussion 904

23. Giordano FA, Welzel G, Abo-Madyan Y, Wenz F: Potential toxicities of prophylactic cranial irradiation. Translational Lung Cancer Research 2012, 1(4):254-262. 
24. Wen PY, Macdonald DR, Reardon DA, Cloughesy TF, Sorensen AG, Galanis E, Degroot J, Wick W, Gilbert MR, Lassman AB, Tsien C, Mikkelsen T, Wong ET, Chamberlain MC, Stupp R, Lamborn KR, Vogelbaum MA, van den Bent MJ, Chang SM: Updated response assessment criteria for high-grade gliomas: response assessment in neuro-oncology working group. J Clin Oncol 2010, 28(11):1963-1972.

25. Brandsma D, Stalpers L, Taal W, Sminia P, van den Bent MJ: Clinical features, mechanisms, and management of pseudoprogression in malignant gliomas. Lancet Oncol 2008, 9(5):453-461.

26. Marks LB, Yorke ED, Jackson A, Ten Haken RK, Constine LS, Eisbruch A, Bentzen SM, Nam J, Deasy JO: Use of normal tissue complication probability models in the clinic. Int J Radiat Oncol Biol Phys 2010, 76(3 Suppl):S10-S19.

27. Herskind C, Wenz F: Radiobiological comparison of hypofractionated accelerated partial-breast irradiation (APBI) and single-dose intraoperative radiotherapy (IORT) with 50-kV X-rays. Strahlenther Onkol 2010, 186(8):444-451.

28. Schneider F, Clausen S, Jahnke A, Steil V, Bludau F, Sutterlin M, Obertacke U, Wenz F: Radiation protection for an intraoperative $\mathrm{x}$-ray source compared to C-arm fluoroscopy. Z Med Phys 2014, 24(3):243-251.

29. Matsutani M, Nakamura O, Nagashima T, Asai A, Fujimaki T, Tanaka H, Nakamura M, Ueki K, Tanaka Y, Matsuda T: Intra-operative radiation therapy for malignant brain tumors: rationale, method, and treatment results of cerebral glioblastomas. Acta Neurochir (Wien) 1994, 131(1-2):80-90.

30. Sakai N, Yamada H, Andoh T, Takada M, Hirata T, Funakoshi T, Doi H, Yanagawa S: Intraoperative radiation therapy for malignant glioma. Neurol Med Chir (Tokyo) 1989, 29(4):312-318.

31. Fujiwara T, Honma Y, Ogawa T, Irie K, Kuyama H, Nagao S, Takashima H, Hosokawa A, Ohkawa M, Tanabe M: Intraoperative radiotherapy for gliomas. J Neurooncol 1995, 23(1):81-86.

32. Shibamoto $\mathrm{Y}$, Yamashita J, Takahashi M, Abe M: Intraoperative radiation therapy for brain tumors with emphasis on retreatment for recurrence following full-dose external beam irradiation. Am J Clin Oncol 1994, 17(5):396-399.

33. Ortiz de Urbina D, Santos M, Garcia-Berrocal I, Bustos JC, Samblas J, Gutierrez-Diaz JA, Delgado JM, Donckaster G, Calvo FA: Intraoperative radiation therapy in malignant glioma: early clinical results. Neurol Res 1995, 17(4):289-294.

34. Nemoto K, Ogawa Y, Matsushita H, Takeda K, Takai Y, Yamada S, Kumabe T: Intraoperative radiation therapy (IORT) for previously untreated malignant gliomas. BMC Cancer 2002, 2:1.

35. Wagner W, Schuller P, Willich N, Schober O, Palkovic S, Morgenroth C, Bartenstein P, Prott FJ, Niewohner U: Intraoperative radiotherapy (IORT) in malignant brain tumors. Strahlenther Onkol 1995, 171(3):154-164.

36. Gouda J, Brown J, Carter D, Dobelbower RR Jr: Malignant brain tumors treated with IORT. Front Radiat Ther Oncol 1997, 31:87-91.

37. Sakai N, Yamada H, Andoh T, Hirata T, Nishimura Y, Miwa Y, Shimizu K, Yanagawa S: Intraoperative radiation therapy for malignant glioma. Neurol Med Chir (Tokyo) 1991, 31(11):702-707.

38. Souhami L, Seiferheld W, Brachman D, Podgorsak EB, Werner-Wasik M, Lustig R, Schultz CJ, Sause W, Okunieff P, Buckner J, Zamorano L, Mehta MP, Curran WJ Jr: Randomized comparison of stereotactic radiosurgery followed by conventional radiotherapy with carmustine to conventional radiotherapy with carmustine for patients with glioblastoma multiforme: report of Radiation Therapy Oncology Group 93-05 protocol. Int J Radiat Oncol Biol Phys 2004, 60(3):853-860.

39. Burnet NG, Jena R, Jefferies SJ, Stenning SP, Kirkby NF: Mathematical modelling of survival of glioblastoma patients suggests a role for radiotherapy dose escalation and predicts poorer outcome after delay to start treatment. Clin Oncol (R Coll Radiol) 2006, 18(2):93-103.

40. Do V, Gebski V, Barton MB: The effect of waiting for radiotherapy for grade III/IV gliomas. Radiother Oncol 2000, 57(2):131-136.

41. Walker MD, Strike TA, Sheline GE: An analysis of dose-effect relationship in the radiotherapy of malignant gliomas. Int J Radiat Oncol Biol Phys 1979, 5(10):1725-1731.

42. Bleehen NM, Stenning SP: A Medical Research Council trial of two radiotherapy doses in the treatment of grades 3 and 4 astrocytoma. The Medical Research Council Brain Tumour Working Party. Br J Cancer 1991, 64(4):769-774

43. Tsien C, Moughan J, Michalski JM, Gilbert MR, Purdy J, Simpson J, Kresel JJ, Curran WJ, Diaz A, Mehta MP: Phase I three-dimensional conformal radiation dose escalation study in newly diagnosed glioblastoma: Radiation Therapy Oncology Group Trial 98-03. Int J Radiat Oncol Biol Phys 2009, 73(3):699-708

44. Tsien Cl, Brown D, Normolle D, Schipper M, Piert M, Junck L, Heth J, Gomez-Hassan D, Ten Haken RK, Chenevert T, et al: Concurrent temozolomide and dose-escalated intensity-modulated radiation therapy in newly diagnosed glioblastoma. Clin Cancer Res 2012, 18(1):273-279.

45. Fisher B, Gunduz N, Coyle J, Rudock C, Saffer E: Presence of a growthstimulating factor in serum following primary tumor removal in mice. Cancer Res 1989, 49(8):1996-2001.

46. Tsuchiya Y, Sawada S, Yoshioka I, Ohashi Y, Matsuo M, Harimaya Y, Tsukada K, Saiki I: Increased surgical stress promotes tumor metastasis. Surgery 2003, 133(5):547-555.

47. Belletti B, Vaidya JS, D'Andrea S, Entschladen F, Roncadin M, Lovat F, Berton S, Perin T, Candiani E, Reccanello S, et al: Targeted intraoperative radiotherapy impairs the stimulation of breast cancer cell proliferation and invasion caused by surgical wounding. Clin Cancer Res 2008, 14(5):1325-1332

48. Goodman JC, Van M, Gopinath SP, Robertson CS: Pro-inflammatory and pro-apoptotic elements of the neuroinflammatory response are activated in traumatic brain injury. Acta Neurochir Suppl 2008, 102:437-439.

49. Hutchinson PJ, O'Connell MT, Rothwell NJ, Hopkins SJ, Nortje J, Carpenter KL, Timofeev I, Al-Rawi PG, Menon DK, Pickard JD: Inflammation in human brain injury: intracerebral concentrations of IL-1alpha, IL-1beta, and their endogenous inhibitor IL-1ra. J Neurotrauma 2007, 24(10):1545-1557.

50. Schultzberg M, Lindberg C, Aronsson AF, Hjorth E, Spulber SD, Oprica M: Inflammation in the nervous system-physiological and pathophysiological aspects. Physiol Behav 2007, 92(1-2):121-128.

51. Gonzalez J, Kumar AJ, Conrad CA, Levin VA: Effect of bevacizumab on radiation necrosis of the brain. Int J Radiat Oncol Biol Phys 2007, 67(2):323-326.

52. Emami B, Lyman J, Brown A, Coia L, Goitein M, Munzenrider JE, Shank B, Solin LJ, Wesson M: Tolerance of normal tissue to therapeutic irradiation. Int J Radiat Oncol Biol Phys 1991, 21(1):109-122.

53. Herskind C, Steil V, Kraus-Tiefenbacher U, Wenz F: Radiobiological aspects of intraoperative radiotherapy (IORT) with isotropic low-energy $X$ rays for early-stage breast cancer. Radiat Res 2005, 163(2):208-215.

54. Chamberlain MC, Glantz MJ, Chalmers L, Van Horn A, Sloan AE: Early necrosis following concurrent Temodar and radiotherapy in patients with glioblastoma. J Neurooncol 2007, 82(1):81-83.

55. Wedge SR, Porteous JK, Glaser MG, Marcus K, Newlands ES: In vitro evaluation of temozolomide combined with X-irradiation. Anticancer Drugs 1997, 8(1):92-97.

56. van Rijn J, Heimans JJ, van den Berg J, van der Valk P, Slotman BJ: Survival of human glioma cells treated with various combination of temozolomide and X-rays. Int J Radiat Oncol Biol Phys 2000, 47(3):779-784.

doi:10.1186/1471-2407-14-992

Cite this article as: Giordano et al:: INTRAGO: intraoperative radiotherapy in glioblastoma multiforme - a Phase I/II dose escalation study. BMC Cancer 2014 15:992.

\section{Submit your next manuscript to BioMed Central and take full advantage of:}

- Convenient online submission

- Thorough peer review

- No space constraints or color figure charges

- Immediate publication on acceptance

- Inclusion in PubMed, CAS, Scopus and Google Scholar

- Research which is freely available for redistribution 